Pacific Journal of Mathematics

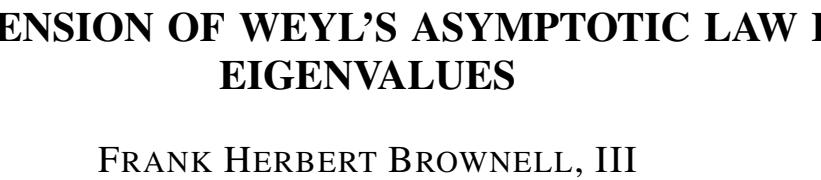




\title{
AN EXTENSION OF WEYL'S ASYMPTOTIC LAW FOR EIGENVALUES
}

\author{
F. H. BROWNE LL
}

1. Introduction. Let $D$ be a bounded, open, connected subset of the plane $E_{2}$ whose boundary $B=\bar{D}-D$ is a simple closed curve whose curvature exists everywhere and is continuous with respect to arc length; consider the eigenvalues $\lambda=\lambda_{n}>0$ of the problem

$$
\nabla^{2} u+\lambda u=0 \text { on } D, \quad u=0 \text { on } B,
$$

where $u(\mathbf{x})$ is to be continuous over $\bar{D}$ and have continuous second partials over $D, \nabla^{2}$ being the Laplacian. It has long been known (see [7, bibliography]) that in this situation, with $0<\lambda_{n} \leq \lambda_{n+1}$ repeated according to multiplicity, the asymptotic distribution of $\lambda_{n}$ is given by Weyl's law

$$
N(t)=\sum_{\lambda_{n} \leq t} 1=\frac{\mu_{2}(D)}{4 \pi} t+o(t)
$$<smiles>[3H][13CH3]</smiles>

where $\mu_{2}(D)$ is the two dimensional Lebesgue measure of $D$. This can be obtained by Tauberian theorems from the estimate

$$
\sum_{n=1}^{\infty} \frac{1}{\lambda_{n}\left(\lambda_{n}+\omega\right)}=\frac{\mu_{2}(D)}{4 \pi} \frac{\ln \omega}{\omega}+\frac{C}{\omega}+O\left(\omega^{-5 / 4}\right), \quad \omega \rightarrow+\infty
$$

(see Carleman [2] for the $E_{3}$ analogue). By domain comparison methods [3, p. 386] Courant has shown that $o(t)$ in $(1.2)$ can be replaced by $O(\sqrt{t} \ln t)$.

In a recent paper $[6$, p. 177 , equation 16$]$ Pleijel replaces the estimate (1.3) by the very much stronger

$$
\sum_{n=1}^{\infty} \frac{1}{\lambda_{n}\left(\lambda_{n}+\omega\right)}=\frac{\mu_{2}(D)}{4 \pi} \frac{\ln \omega}{\omega}+\frac{C}{\omega}+\frac{l(B)}{8} \frac{1}{\omega^{3 / 2}}+O\left(\frac{1}{\omega^{2}}\right)
$$

over $\omega \geq 1$ in case the curve $B$ is very smooth (that is, it has an infinitely

Received Octoher 19, 1953.

Pacific J. Math. 5 (1955), 483-499 
differentiable parametric representation), where $C$ is an unknown real constant and $l(B)$ is the total length of $B$. Pleijel's estimate (1.4) follows easily from a deep investigation jointly with $\mathrm{M}$. T. Ganelius on the compensating part of the Green function, as yet unpublished. This investigation uses integral equations over the boundary $B$, while estimates like (1.3) come from a simple application of the maximum principle over $D$ to the modified Green function. Pleijel suggests it should be possible to sharpen (1.2) by using his methods to investigate the analogue of (1.4) over complex $\omega$.

It is the purpose of this paper to show that from (1.4) alone we can replace (1.2) by

$$
N(t)=\frac{\mu_{2}(D)}{4 \pi} t-\frac{l(B)}{4 \pi} t^{1 / 2}+\widetilde{O}(\ln t)
$$

in a certain sense. Precisely our result $(2.13)$ is that with

$$
F(t)=N(t)-\left\{\frac{\mu_{2}(D)}{4 \pi} t-\frac{l(B)}{4 \pi} t^{1 / 2}\right\}
$$

we have

$$
\left|\int_{1 / 2 \lambda_{1}}^{\infty} \exp \left(-\frac{\rho^{2}}{2}\left(\ln \frac{u}{t}\right)^{2}\right) d F(t)\right| \leq\left[M \exp \left(\frac{\pi^{2} \rho^{2}}{2}+\frac{1}{2 \rho^{2}}\right)\right] \ln u
$$

over all real $u \geq e$ and all $\rho>0$ for some $M<+\infty$. Moreover, if $N(t)$ has an ordinary asymptotic series in powers of $t$, it must be consistent with (1.5). We discuss briefly the possibility of sharpening (1.5) by replacing averaged $\widetilde{O}$ estimates by ordinary ones. We also note the utility of our consistency result in proving false a conjecture of Minakshisundaram [5, p. 331, no. 2] about the asymptotic behavior of $N(t)$.

Clearly our theorems will apply to give results like (1.5) for a wide variety of more general problems than (1.1) for which estimates like (1.4) obtain; in particular such results hold for $(1.1)$ in 3 -space $E_{3}$.

2. Results and proofs. The difficulty arising in trying to get an asymptotic series like (1.5), with $\widetilde{O}$ replaced by an ordinary $O$ or $o$, is that Tauberian theorems yielding such results seem to require essential nonnegative conditions after subtracting all but the last term of the series. It is quite clear that $N(t)$ does not satisfy such a condition. For this reason we use an indirect 
Abelian type argument $[4$, p. 224] to get averaged error estimates of the $\widetilde{O}$ type. The two first theorems here establish the significance of these averaged error estimates, which despite the resemblance to Gaussian summability seem to be little used for asymptotic series. Cramér [1, p. 819 and p. 823, (3)] has used Caesaro-1 type averaged error estimates on lattice point problems, but such processes do not appear strong enough for use here.

Throughout the paper all integrals are to be understood in the Lebesgue or Lebesgue-Stieltjes sense, and for the following two theorems it is understood that $F(t)$ is to be real valued of bounded variation over every finite interval of $[0, \infty)$, with positive $b$ a continuity point of $F(t)$. Also $|d F(t)|$ stands for $d V_{F}(t)$ where $V_{F}(t)$ is the total variation of $F$ over $[b, t]$.

THEOREM 1. If

$$
\int_{b}^{\infty} t^{-r_{0}}|d F(t)|<+\infty
$$

for some $r_{0}>0$, if

$$
\psi(s)=\int_{b}^{\infty} t^{-s} d F(t)
$$

which must exist and be analytic in $s$ over $K[s]>r_{0}$, also has an analytic continuation without singularities throughout $R[s]>0$, and if

$$
|\psi(r+i v)| \leq \frac{M_{1}}{r} e^{h|v|}
$$

over $0<r \leq r_{0}$ and all real $v$ for some $M_{1}<+\infty$ and $h \geq 0$, then over all real $u \geq e$ and $\rho>0$ we have

$$
\left|\int_{b}^{\infty} e^{-\left(\rho^{2} / 2\right)(\ln (u / t))^{2}} d F(t)\right| \leq\left(2 M_{1} \exp \left(1+\frac{\rho^{2}}{2} h^{2}+\frac{1}{2 \rho^{2}}\right)\right) \ln u .
$$

In view of (2.1) it becomes convenient to define $F(t)=\widetilde{O}(f(t))$ over $t \geq b$ for some nonnegative $f(t)$ defined over $t \geq k>0$ if for each $\rho>0$ there exists some $M_{\rho}<* \infty$ such that the left side of $(2.1)$ exists and is $\leq M_{\rho} f(u)$ for all $u \geq k$. With this definition we can restate the conclusion of Theorem 1 as

$$
F(t)=\widetilde{O}(\ln t)
$$


over $t \geq b$ with $k=e$. Note that in (2.1) $M_{\rho} \rightarrow+\infty$ as either $\rho \rightarrow 0^{+}$or $\rho \longrightarrow+\infty$, so that $(2.1)$ becomes meaningless then. The significance of the result $(2.1)$ is greatly increased by the following consistency the orem.

TheOREM 2. If

$$
\int_{b}^{\infty} t^{-r_{0}}|d F(t)|<+\infty
$$

for some $r_{0}>0$, if

$$
F(t)=\widetilde{O}(\ln t)
$$

over $t \geq b$, and if

$$
F(t)=c_{1} t^{r_{1}}+o\left(t^{r_{1}}\right)
$$

as $t \longrightarrow+\infty$ for some $r_{1}>0$, then $c_{1}=0$.

Proof of Theorem 1. Let

$$
f_{z}(y)=\exp \left(-\frac{\rho^{2}}{2} y^{2}-z y\right)
$$

for $\rho>0$ and any complex $z$; thus

$$
\frac{1}{\sqrt{2 \pi}} \int_{-\infty}^{\infty} f_{z}(y) e^{-i v y} d y=\frac{1}{\rho} \exp \left(-\frac{1}{2 \rho^{2}}\left(v+\frac{z}{i}\right)^{2}\right)
$$

Now

$$
M \geq \int_{b}^{\tau} t^{-r_{0}}|d F(t)| \geq \tau^{-r_{0}} V_{F}(\tau)
$$

shows

$$
V_{F}(t)=O\left(t^{r_{0}}\right)
$$

thus

$$
g(z, \omega)=\int_{y=\ln b}^{\infty} f_{z}(\omega-y) e^{-z y} d F\left(e^{y}\right)
$$


exists as an entire function of $z$ over all real $\omega$ and all complex $z$. The Fubini theorem also shows $g(z, \omega) \in L_{1}(-\infty, \infty)$ over $\omega$ with

$$
\frac{1}{\sqrt{2 \pi}} \int_{-\infty}^{\infty} g(z, \omega) e^{-i v \omega} d \omega=\frac{1}{\rho} \exp \left(-\frac{1}{2 \rho^{2}}\left(v+\frac{z}{i}\right)^{2}\right) \psi(z+i v)
$$

over $R[z+i v]=R[z] \geq r_{0}, v$ being real. But the right side of $(2.2)$ is in $L_{1}(-\infty, \infty)$ over $v$ since

$$
|\psi(s)| \leq \int_{b}^{\infty} t^{-r}|d F(t)|, \quad r=R[s]
$$

and thus the Fourier transform inverse yields

$$
\begin{aligned}
\int_{y=\ln b}^{\infty} f_{z}(\omega-y) e^{-z y} d F\left(e^{y}\right)=g(z, \omega) \\
=\frac{1}{\sqrt{2 \pi}} \int_{-\infty}^{\infty} \exp \left(-\frac{(v+z / i)^{2}}{2 \rho^{2}}\right) \psi(z+i v) e^{i v \omega} \frac{d v}{\rho}
\end{aligned}
$$

for $R[z] \geq r_{0}$. The given estimate on $\psi(s)$ actually makes the far right side of (2.3) exist and be analytic in $z$ throughout $R[z]>0$, and thus by analytic continuation (2.3) holds there also. Thus with $z=r$ we have for every positive $r$ and $\rho$ and for every real $\omega$ the estimate

$$
\begin{aligned}
\left|\int_{y=\ln b}^{\infty} \exp \left(-\frac{\rho^{2}}{2}(\omega-y)^{2}-r \omega\right) d F\left(e^{y}\right)\right| \\
\leq \frac{1}{\sqrt{2 \pi}} \int_{-\infty}^{\infty} \exp \left(\frac{-v^{2}+r^{2}}{2 \rho^{2}}\right) \frac{M_{1}}{r} e^{h|v| \frac{d v}{\rho}} \\
\quad \frac{M_{1}}{r} \exp \left(\frac{r^{2}}{2 \rho^{2}}+\frac{h^{2} \rho^{2}}{2}\right)\left\{\frac{1}{\sqrt{2 \pi}} \int_{0}^{\infty} e^{-1 / 2(v / \rho-\rho h)^{2}} \frac{d v}{\rho}\right. \\
\left.\quad+\frac{1}{\sqrt{2 \pi}} \int_{-\infty}^{0} e^{-1 / 2(v / \rho+\rho h)^{2}} \frac{d v}{\rho}\right\} \leq \frac{2 M_{1}}{r} \exp \left(\frac{r^{2}}{2 \rho^{2}}+\frac{h^{2} \rho^{2}}{2}\right) .
\end{aligned}
$$

Multiplying (2.4) by $e^{r \omega}$ and letting $r=1 / \omega>0$ we note for $\omega \geq 1$ that 


$$
r \omega+\frac{r^{2}}{2 \rho^{2}}-\ln r=1+\frac{1}{2 \rho^{2} \omega^{2}}+\ln \omega \leq 1+\frac{1}{2 \rho^{2}}+\ln \omega,
$$

thus with $y=\ln t$ and $\omega=\ln u \geq 1$ we get the estimate (2.1) as desired.

Proof of Theorem 2. As before we have

$$
|F(t)| \leq|F(b)|+V_{F}(t)=O\left(t^{r_{0}}\right),
$$

so that we can integrate by parts in the left side of $(2.1)$ and obtain from $F(t)=\widetilde{O}(\ln t)$ over $t \geq b$ the estimate

$$
\left|\rho^{2} \int_{y=\ln b}^{\infty} F\left(e^{y}\right)(\omega-y) e^{-\rho^{2}(\omega-y)^{2} / 2} d y\right| \leq|F(b)|+M_{\rho} \omega
$$

over $\omega \geq k>0$. Now we are given

$$
F(t)=c_{1} t^{r_{1}}+f(t) t^{r_{1}}
$$

over $t \geq b$ with $\lim _{t \rightarrow+\infty} f(t)=0$. Thus multiplying (2.5) by $e^{-r_{1} \omega}$, letting $y=\omega-x$, and taking $\omega \longrightarrow+\infty$ we get

$$
\begin{aligned}
0= & \lim _{\omega \rightarrow+\infty}\left\{c_{1} \int_{-\infty}^{\omega-\ln b} x \exp \left(-r_{1} x-\frac{\rho^{2}}{2} x^{2}\right) d x\right. \\
& \left.+\int_{-\infty}^{\omega-\ln b} f\left(e^{\omega-x}\right) x \exp \left(-r_{1} x-\frac{\rho^{2}}{2} x^{2}\right) d x\right\} .
\end{aligned}
$$

Defining $f(t)=0$ for $t<b$ we obtain

$$
\begin{aligned}
0=c_{1} & \int_{-\infty}^{\infty} x \exp \left(-r_{1} x-\frac{\rho^{2}}{2} x^{2}\right) d x \\
& +\lim _{\omega \rightarrow+\infty}\left\{\int_{-\infty}^{\infty} f\left(e^{\omega-x}\right) x \exp \left(-r_{1} x-\frac{\rho^{2}}{2} x^{2}\right) d x\right\} .
\end{aligned}
$$

$f(t)$ being bounded over all real $t$ since $\lim _{t \rightarrow+\infty} f(t)=0$, and thus also $\lim _{\omega \rightarrow+\infty} f\left(e^{\omega-x}\right)=0$, dominated convergence applied to (2.6) yields 


$$
0=c_{1} \int_{-\infty}^{\infty} x \exp \left(-r_{1} x-\frac{\rho^{2}}{2} x^{2}\right) d x
$$

But

$$
\int_{-\infty}^{\infty} x \exp \left(-r_{1} x-\frac{\rho^{2}}{2} x^{2}\right) d x=\int_{0}^{\infty} x\left(e^{-r_{1} x}-e^{r_{1} x}\right) e^{-\rho^{2} x^{2} / 2} d x<0
$$

for $r_{1}>0$, so that $c_{1}=0$ follows.

To apply these two theorems we use a standard contour integral transformation on Pleijel's estimate (1.4). The contour $C_{\rho}, \rho \geq 0$, in the $z$ plane is defined to be first along the negative real axis from $-\propto$ to $-\rho$, then around the circle $z=\rho e^{i \theta}$ from $\theta=-\pi$ to $\theta=\pi$, then back along the axis to $-\infty$. On this contour we define

$$
(z)_{c}^{s-1}=|z|^{s-1} e^{i(s-1) \theta}, \quad z=|z| e^{i \theta}
$$

with $\theta=-\pi,-\pi<\theta<\pi, \theta=\pi$ on the three parts respectively. The well known results are formulated in the following two lemmas (Carleman [2]), and we sketch the proofs for the sake of completeness.

LEMMA 3. If $0<\lambda_{n} \leq \lambda_{n+1}, a_{n}$ real, if

$$
\sum_{n=1}^{\infty} \frac{\left|a_{n}\right|}{\lambda_{n}^{2}}<+\infty
$$

and if

$$
\sum_{n=1}^{\infty} \frac{1}{\lambda_{n}^{2}}<+\infty
$$

then

$$
h(z)=\sum_{n=1}^{\infty} \frac{a_{n}}{\lambda_{n}\left(\lambda_{n}-z\right)}
$$

converges absolutely and is analytic in all complex $z$ except for simple poles at each $\lambda_{n}$. Moreover, for $0<\rho<\lambda_{1}$ the function 


$$
\frac{1}{2 \pi i} \int_{C_{\rho}} h(z) \frac{d z}{(z)_{c}^{s-1}}
$$

exists and is analytic in $s$ over $R[s]>2$,

$$
\sum_{n=1}^{\infty} a_{n} \lambda_{n}^{-s}
$$

converges absolutely and uniformly over $R[s] \geq 2$, and over $R[s]>2$ we obtain

$$
\sum_{n=1}^{\infty} a_{n} \lambda_{n}^{-s}=\frac{1}{2 \pi i} \int_{C_{\rho}} h(z) \frac{d z}{(z)_{c}^{s-1}}
$$

LEMMA 4. If the assumptions of Lemma 3 are satisfied and if

$$
h(-\omega)=\sum_{p=1}^{k} \frac{m_{p}+l_{p} \ln \omega}{\omega^{2-r_{p}}}+O\left(\frac{1}{\omega^{2}}\right)
$$

holds over $\omega \geq 1$ with $0<r_{k}<r_{k-1}<\cdots<r_{1}<2$, then

$$
g(s)=\sum_{k=1}^{\infty} a_{n} \lambda_{n}^{-s}
$$

has an analytic extension into $R[s]>0$ except for poles at $r_{p}$,

$$
\begin{aligned}
g(s)= & g_{k}(s)+\sum_{p=1}^{k}\left\{l_{p} \frac{\sin \pi\left(r_{p}-1\right)}{\pi} \frac{1}{\left(s-r_{p}\right)^{2}}\right. \\
& \left.+\left[m_{p} \frac{\sin \pi\left(r_{p}-1\right)}{\pi}+l_{p} \cos \pi\left(r_{p}-1\right)\right] \frac{1}{s-r_{p}}\right\}
\end{aligned}
$$

with $g_{k}(s)$ analytic in $s$ throughout $R[s]>0$, and

$$
\left|g_{k}(r+i v)\right| \leq \frac{M_{2}}{r} e^{\pi|v|}
$$

over $0<r \leq 2$ and all real $v$ for some $M_{2}<+\infty$. 
We remark that $r_{1}<2$ is no real restriction in (2.8), since the assumptions of Lemma 3 imply $\lim _{\omega \rightarrow+\infty} h(-\omega)=0$. In demonstrating Lemma 3, the stated analyticity of $h(z)$ is clear as well as

$$
|h(z)| \leq\left(\inf _{j \geq 1}\left|1-\frac{z}{\lambda_{j}}\right|\right)^{-1}\left(\sum_{n=1}^{\infty} \frac{\left|a_{n}\right|}{\lambda_{n}^{2}}\right),
$$

so

$$
g(s)=\frac{1}{2 \pi i} \int_{C_{\rho}} h(z) \frac{d z}{(z)_{c}^{s-1}}
$$

exists and is analytic in $s$ over $R[s]>2$. To show

$$
g(s)=\sum_{n=1}^{\infty} a_{n} \lambda_{n}^{-s}
$$

there for (2.7), let $C_{m}$ be the vertical line contour from $x_{m}-i \infty$ to $x_{m}+i \infty$ for $x_{m}$ with $\lambda_{n-1}<x_{m}<\lambda_{n}$, so that using the estimate on $h(z)$ to shift from $C_{\rho}$ to $C_{m}$ we obtain

$$
g(s)-\sum_{j=1}^{n-1} a_{j} \lambda_{j}^{-s}=\frac{1}{2 \pi i} \int_{C_{m}} h(z) \frac{d z}{z^{s-1}}
$$

for $R[s]>2, h(z)$ having the residue

$$
-\lambda^{-1}\left(\sum_{\lambda_{j}=\lambda} a_{j}\right)
$$

at $\lambda$.

To pass from $(2.10)$ to $(2.7)$, note that

$$
\left[\limsup _{n \rightarrow \infty} \lambda_{n}^{2}\left(\lambda_{n}-\lambda_{n-1}\right)\right]=+\infty,
$$

since otherwise

$$
\lambda_{n}-\lambda_{1}=\sum_{j=2}^{n}\left(\lambda_{j}-\lambda_{j-1}\right)
$$


would be bounded by

$$
M\left(\sum_{j=1}^{\infty} \frac{1}{\lambda_{j}^{2}}\right)
$$

which contradicts $\lambda_{n} \longrightarrow+\infty$ and therefore contradicts

$$
\sum_{n=1}^{\infty} \frac{1}{\lambda_{n}^{2}}<+\infty
$$

Thus there exists a sequence $n_{m}$ such that

$$
n_{m}<n_{m+1}, \lambda_{n}-\lambda_{n-1}>0 \text {, and } \lambda_{n}^{2}\left(\lambda_{n}-\lambda_{n-1}\right) \longrightarrow+\infty \text { as } m \longrightarrow+\infty \text { for } n=n_{m} \text {. }
$$

We choose

$$
x_{m}=\left\{\max \left(\lambda_{n}+\lambda_{n-1}\right) / 2, \quad\left(\lambda_{n}-1\right)\right\}
$$

for $n=n_{m}$, so that

$$
\frac{\lambda_{n}}{x_{m}} \leq 1+\frac{1}{x_{m}} \rightarrow 1
$$

and

$$
\frac{x_{m}}{\lambda_{n}-x_{m}} \leq x_{m}+\frac{2 x_{m}}{\lambda_{n}-\lambda_{n-1}}=x_{m}+2 x_{m}^{3}\left(\frac{\lambda_{n}}{x_{m}}\right)^{2} \frac{1}{\lambda_{n}^{2}\left(\lambda_{n}-\lambda_{n-1}\right)}=O\left(x_{m}^{3}\right) .
$$

With $z=x_{m}+i t$ and $s=r+i v, r>2$, clearly

$$
|h(z)| \leq \frac{M}{\left|1-\left(x_{m}+i t\right) / \lambda_{n}\right|}
$$

and

$$
\left|\frac{1}{z^{s-1}}\right| \leq \frac{\exp (\pi|v| / 2)}{|z|^{r-1}}
$$

with $L(v)=M \exp (\pi|v| / 2)$ make 


$$
\begin{aligned}
& \left|h(z) \frac{1}{z^{s-1}}\right| \leq\left(x_{m}\right)^{2-r}\left(\frac{\lambda_{n}}{x_{m}}\right) \frac{L(v)}{\lambda_{n}-x_{m}} \quad \text { over }|t| \leq \lambda_{n}-x_{m}, \\
& \left|h(z) \frac{1}{z^{s-1}}\right| \leq\left(x_{m}\right)^{2-r}\left(\frac{\lambda_{n}}{x_{m}}\right) \frac{L(v)}{|t|} \quad \text { over } \lambda_{n}-x_{m} \leq|t| \leq x_{m},
\end{aligned}
$$

and

$$
\left|h(z) \frac{1}{z^{s-1}}\right| \leq\left(\frac{\lambda_{n}}{x_{m}}\right) \frac{L(v)}{|t|^{r-1}}
$$

over $x_{m} \leq|t|$.

Thus integrating over these respective parts of $C_{m}$, and using

$$
\ln \left(\frac{x_{m}}{\lambda_{n}-x_{m}}\right)=O\left(\ln x_{m}\right)
$$

the right side of $(2.10) \longrightarrow 0$ as $m \longrightarrow+\infty$ and (2.7) follows.

Passing to Lemma 4, from the estimate $(2.8)$ it is clear that

$$
g(s)=\frac{1}{2 \pi i} \int_{C_{\rho}} h(z) \frac{d z}{(z)_{c}^{s-1}}
$$

extends analytically from $R[s]>2$ to $R[s]>r_{1}$. Also for $r_{1}<R[s]<2$, $C_{\rho}$ can be shifted to $C_{0}$ yielding

$$
g(s)=\frac{1}{2 \pi i} \int_{C_{0}} h(z) \frac{d z}{(z)_{c}^{s-1}}=\frac{\sin \pi(s-1)}{\pi} \int_{0}^{\infty} h(-\omega) \frac{d \omega}{\omega^{s-1}} .
$$

Now here

$$
\frac{\sin \pi(s-1)}{\pi} \int_{0}^{1} h(-\omega) \frac{d \omega}{\omega^{s-1}}=\frac{\sin \pi(s-1)}{-\pi(s-2)}\left\{h(-1)+\int_{0}^{1} h^{\prime}(-\omega) \frac{d \omega}{\omega^{s-2}}\right\},
$$

which is analytic in $s$ over $R[s]<3$, having a removable singularity at $s=2$. Also

$$
\begin{aligned}
& \frac{\sin \pi(s-1)}{\pi} \int_{1}^{\infty} \omega^{r-2} \frac{d \omega}{\omega^{s-1}}=\frac{\sin \pi(s-1)}{\pi(s-r)} \text { and } \\
& \frac{\sin \pi(s-1)}{\pi} \int_{1}^{\infty} \omega^{r-2} \ln \omega \frac{d \omega}{\omega^{s-1}}=\frac{\sin \pi(s-1)}{\pi(s-r)^{2}}
\end{aligned}
$$


with principal parts

$$
\frac{\sin \pi(r-1)}{\pi(s-r)} \text { and } \frac{\sin \pi(r-1)}{\pi(s-r)^{2}}+\frac{\cos \pi(r-1)}{s-r}
$$

respectively at $s=r$. Thus (2.9) clearly follows from (2.8) and (2.11). Also from

$$
|\sin \pi(s-1)| \leq 2 e^{\pi|v|} \text { and } \int_{1}^{\infty} \frac{1}{\omega^{2}} \frac{d \omega}{\omega^{r-1}}=\frac{1}{r}
$$

the stated estimate for $g_{k}(s)$ follows.

We combine Lemma 4 with our two previous theorems to obtain the following result.

THE OREM 5. If the assumptions of Lemma 4 are satisfied with

$$
l_{p} \sin \left(\pi r_{p}\right)=0
$$

in (2.8), then

$$
H(t)=\sum_{\lambda_{n} \leq t} a_{n}
$$

satisfies

$$
H(t)=\left\{\sum_{p=1}^{k} \frac{t^{r_{p}}}{r_{p}}\left(-m_{p} \frac{\sin \pi r_{p}}{\pi}-l_{p} \cos \pi r_{p}\right)\right\}+\widetilde{O}(\ln t)
$$

over $t \geq b$ where $0<b<\lambda_{1}$. Furthermore, if $H(t)$ has an ordinary asymptotic series in powers of $t$ as $t \longrightarrow+\infty$, such a series must coincide term for term as far as it goes with the terms of (2.12).

Proof. Let

$$
F(t)=H(t)-\left\{\sum_{p=1}^{k} \frac{t^{r_{p}}}{r_{p}}\left(-m_{p} \frac{\sin \pi r_{p}}{\pi}-l_{p} \cos \pi r_{p}\right)\right\},
$$

and note that 


$$
\int_{b}^{\infty} t^{-s} d\left(\frac{t^{r}}{r}\right)=\int_{b}^{\infty} t^{r-s-1} d t=\frac{b^{r-s}}{s-r}
$$

for $R[s]>r$ and $b>0$. Also with $0<b<\lambda_{1}$, we have

$$
\int_{b}^{\infty} t^{-s} d H(t)=\sum_{n=1}^{\infty} a_{n} \lambda_{n}^{-s}
$$

for $R[s] \geq 2$. Thus from Lemma 4 we see that

$$
\psi(s)=\int_{b}^{\infty} t^{-s} d F(t)
$$

has an analytic continuation without singularities into $R[s]>0$ by the cancellation of principal parts at each $r_{p}=s$. Also the conditions of Theorem 1 are satisfied with $r_{0}=2$ and $h=\pi$; thus (2.1) yields (2.12). Theorem 2 gives the consistency statement obviously.

To apply this theorem to our problem (1.1), we remark that the desired condition

$$
\sum_{n=1}^{\infty} \frac{1}{\lambda_{n}^{2}}<+\infty
$$

follows from Green's function being in $L_{2}(D \times D)$, and thus a Hilbert-Schmidt kernel. Thus Pleijel's estimate (1.4) yields (2.12) with

$$
\begin{gathered}
k=2, r_{1}=1, m_{1}=C, l_{1}=\frac{\mu_{2}(D)}{4 \pi}, \sin \left(\pi r_{1}\right)=0, \cos \left(\pi r_{1}\right)=-1, \\
r_{2}=\frac{1}{2}, m_{2}=\frac{l(B)}{8}, \quad l_{2}=0, \sin \left(\pi r_{2}\right)=1,
\end{gathered}
$$

and we can state the following.

Corollary 6. Let the open, bounded, connected set $D$ in the plane $E_{2}$ have its boundary $B$ an infinitely differentiable Jordan curve so that Pleijel's estimate (1.4) holds for the problem (1.1). Then over $t \geq \lambda_{1} / 2$ we have

$$
N(t)=\sum_{\lambda_{n} \leq t} 1=\frac{\mu_{2}(D)}{4 \pi} t-\frac{l(B)}{4 \pi} t^{1 / 2}+\widetilde{O}(\ln t)
$$


and as in Theorem 5 any ordinary asymptotic series for $N(t)$ must be consistent vith (2.13).

If we consider the real valued eigenfunction $u_{n}(\mathbf{x})$ of problem $(1.1)$, in

place of (1.4) Pleijel gets [6, equation 6 and second equation of p. 177] over $\mathbf{x} \in D$ and $\omega \geq 1$

$$
\begin{aligned}
\sum_{n=1}^{\infty} \frac{\left|u_{n}(\mathbf{x})\right|^{2}}{\lambda_{n}\left(\lambda_{n}+\omega\right)}=\frac{1}{4 \pi} \frac{\ln \omega}{\omega}+\frac{C(\mathbf{x})}{\omega} & +\frac{1}{2 \pi} \frac{K_{0}(2 r(\mathbf{x}) \sqrt{w})}{\omega} \\
& +O\left(\frac{e^{-2 A r(\mathbf{x}) \sqrt{w}}}{w^{3 / 2}}\right),
\end{aligned}
$$

$A>0, r(\mathbf{x})$ the distance from $\mathbf{x} \in D$ to $B$, the $O$ symbol being uniform over $\mathbf{x} \in D$ as well as $\omega \geq 1$. Now $K_{0}(r)$, the modified Bessel function of the second kind and zero order, has

$$
K_{0}(r)=\sqrt{\frac{\pi}{2 r}} e^{-r}\left(1+o\left(\frac{1}{r}\right)\right)
$$

as $r \longrightarrow+\infty[8$, p. 374]. Thus for each fixed $\mathbf{x} \in D$, with $r(\mathbf{x})>0$, we have over $\omega \geq 1$

$$
\sum_{n=1}^{\infty} \frac{\left|u_{n}(\mathbf{x})\right|^{2}}{\lambda_{n}\left(\lambda_{n}+\omega\right)}=\frac{1}{4 \pi} \frac{\ln \omega}{\omega}+\frac{C(\mathbf{x})}{\omega}+O \mathbf{x}\left(\frac{1}{\omega^{2}}\right),
$$

where the symbol $O_{\mathbf{x}}$ now depends on $\mathbf{x} \in D$. It is also easy to see that at each $\mathbf{x} \neq \mathbf{y}$ with $\mathbf{x}, \mathbf{y} \in D$ we have over $\omega \geq 1$

$$
\sum_{n=1}^{\infty} \frac{u_{n}(\mathbf{x}) u_{n}(\mathbf{y})}{\lambda_{n}\left(\lambda_{n}+\omega\right)}=\frac{C(\mathbf{x}, \mathbf{y})}{\omega}+O_{\mathbf{x}, \mathbf{y}}\left(\frac{1}{\omega^{2}}\right),
$$

and indeed much better estimates than $O\left(1 / \omega^{2}\right)$ hold in (2.15) and (2.16). Also

$$
\sum_{n=1}^{\infty} \frac{\left|u_{n}(\mathbf{x})\right|^{2}}{\lambda_{n}^{2}}<+\infty
$$

is known at each $\mathbf{x} \in D$; thus Theorem 5 yields the following. 
Corollary 7. Let $D$ be as in Corollary 6, so that (2.15) and (2.16) hold at each $\mathbf{x} \neq \mathbf{y}$ with $\mathbf{x}, \mathbf{y} \in D$. Then over $t \geq \lambda_{1} / 2$

$$
\sum_{\lambda_{n} \leq t}\left|u_{n}(\mathbf{x})\right|^{2}=\frac{1}{4 \pi} t+\widetilde{O}(\ln t), \sum_{\lambda_{n} \leq t} u_{n}(\mathbf{x}) u_{n}(\mathbf{y})=\widetilde{O}(\ln t),
$$

with consistency of these series with ordinary asymptotic series, if any, as in Theorem 5.

3. Discussion of results. It is quite clear that $\widetilde{O}(\ln t)$ in $(2.17)$ can be replaced by much stronger estimates in the $\widetilde{O}$ sense, say $\widetilde{O}(1 / t)$, since much more than $O\left(1 / \omega^{2}\right)$ holds in (2.15) and (2.16). In (2.13) additional terms enter if a stronger $\widetilde{O}$ type error estimate is required. These are due to additional terms entering Pleijel's equation (1.4), one of them involving the mean square curvature of $B$, if $O\left(1 / \omega^{2}\right)$ is replaced by a stronger estimate.

A much more difficult and interesting question is the extent to which the averaged $\widetilde{O}$ estimates in our results may be replaced by ordinary $O$ estimates for the problem (1.1). It is clear that by improving the $O\left(e^{\pi|v|}\right)$ estimate on the analytic continuation of

$$
\sum_{n=1}^{\infty} \lambda_{n}^{-s}, s=r+i v
$$

we can replace the Gauss kernel

$$
\exp \left(-\frac{\rho^{2}}{2}(\omega-y)^{2}\right)
$$

in our definition of $\widetilde{O}$ by less well behaved ones. We could get ordinary $O$ estimates if we could use the characteristic function kernel $\chi_{[-1,1]}(\omega-y)$, but since its Fourier transform is essentially $v^{-1} \sin v$, the analogue of the proof of Theorem 1 would then seem to require stronger conditions on

$$
\sum_{n=1}^{\infty} \lambda_{n}^{-s}
$$

than can be expected to hold.

It is known from the refined results of geometric number theory [1, p. 823] that $M(x)$, defined as the number of integer lattice points $(m, n)$ in the plane satisfying $m^{2}+n^{2} \leq x$, satisfies 


$$
M(x)=\pi x+O\left(x^{1 / 3}\right)
$$

Since

$$
\lambda=\left(n^{2}+m^{2}\right) \frac{\pi^{2}}{b^{2}}
$$$$
n>0, m>0
$$

for the eigenvalues of (1.1) with $D$ a square of side $b$, the eigenfunctions being products of sine functions, we clearly see that

$$
N(t)=\frac{1}{4}\left\{M\left(\frac{b^{2} t}{\pi^{2}}\right)-\left(4\left[\frac{b \sqrt{t}}{\pi}\right]+1\right)\right\}=\frac{b^{2}}{4 \pi} t-\frac{4 b}{4 \pi} \sqrt{t}+O\left(t^{1 / 3}\right)
$$

for square $D, 4[b \sqrt{t} / \pi]+1$ being the number of lattice points on the axes. This asymptotic result for $N(t)$ agrees with (2.13), although the corners of a square prevent it from satisfying the smooth boundary conditions required in Corollary 6. By carelessly dropping the $\sqrt{t}$ term in going from $M(x)$ to $N(t)$, Minakshisundaram [5, p. 331, no. 2] is led to the conjecture that domain comparison methods $[3, \mathrm{p} .386]$ should yield

$$
N(t)=\frac{\mu_{2}(D)}{4 \pi} t+O\left(t^{1 / 3}\right)
$$

for general domains $D$. Clearly the consistency statement of Corollary 6 makes such asymptotic behavior impossible for $N(t)$.

\section{REFERENCES}

1. H. Bohr and H. Cramer, Die neuere Entwicklung der analytischen Zahlentheorie, Encykl. der Math. Wiss., 2, part 3, no. 8.

2. T. Carleman, Propriétés asymptotics des fonctions fondamentales des membranes vibrantes, Förhand. 8th Skand. Mat. Kongress, (1934), 34-44.

3. R. Courant and D. Hilbert, Methoden der mathematischen Physik, vol. 1, Springer, Berlin, 1931.

4. G. Doetsch, Laplace transforms, Springer, Berlin.

5. S. Minakshisundaram, Lattice point and eigenvalue problems, Symposium on Spectral Theory, Stillwater, Okla., (1951), 325-332.

6. A. Pleijel, Sur les valeurs et les fonctions propres des membranes vibrantes, Comm. Sem. Math. Univ. Lund (Medd. Lunds Univ. Mat. Sem.), suppl. (Riesz) vol., (1952), $173-179$. 
7. H. Weyl, Ramifications of the eigenvalue problem, Bull. Amer. Math. Soc., 56 (1950), $115-139$.

8. E. T. Whittaker and G. N. Watson, A course of modern analysis, Cambridge, 1946.

UNIVERSITY OF WASHINGTON AND

INSTITUTE FOR ADVANCED STUdy 



\section{PACIFIC JOURNAL OF MATHEMATICS}

\section{EDITORS}

\section{H.L. ROY DEN}

Stanford University

Stanford, California

E. HEWITT

University of Washington

Seattle 5, Washington

\section{R. P. DILWORTH}

California Institute of Technology

Pasadena 4, California

* Alfred Horn

University of California

Los Angeles 24, California

\section{ASSOCIATE EDITORS}

\begin{abstract}
H. BUSEMANN
HERBERT FEDERER

MARSHALL HALL
\end{abstract}

\section{P.R. HALMOS}

HEINZ HOPF

ALFRED HORN
R.D. JAMES

BØRGE JESSEN

PAUL LÉVY
GEORGE PÓLYA

J.J. STOKER

KOSAKU YOSIDA
UNIVERSITY OF BRITISH COLUMBIA CALIFORNIA INSTITUTE OF TECHNOLOGY UNIVERSITY OF CALIFORNIA, BER KELEY UNIVERSITY OF CALIFORNIA, DAVIS UNIVERSITY OF CALIFORNIA, LOS ANGELES UNIVERSITY OF CALIFORNIA, SANTA BARBARA MONTANA STATE UNIVERSITY

UNIVERSITY OF NEVADA

OREGON STATE COLLEGE

UNIVERSITY OF OREGON
UNIVERSITY OF SOUTHERN CALIFORNIA STANFORD UNIVERSITY UNIVERSITY OF UTAH WASHINGTON STATE COLLEGE UNIVERSITY OF WASHINGTON

AMERICAN MATHEMATICAL SOCIETY HUGHES AIRCRAFT COMPANY SHELL DEVELOPMENT COMPANY

Mathematical papers intended for publication in the Pacific Journal of Mathematics should be typewritten (double spaced), and the author should keep a complete copy. Manuscripts may be sent to any of the editors. Manuscripts intended for the outgoing editors should be sent to their successors. All other communications to the editors should be addressed to the managing editor, Alfred Horn, at the University of California Los Angeles 24, California.

50 reprints of each article are furnished free of charge; additional copies may be obtained at cost in multiples of 50 .

The Pacific Journal of Mathematics is published quarterly, in March, June, September, and December. The price per volume (4 numbers) is $\$ 12.00$; single issues, $\$ 3.50$; back numbers (Volumes $1,2,3$ ) are available at $\$ 2.50$ per copy. Special price to individual faculty members of supporting institutions and to individual members of the American Mathematical Society: $\$ 4.00$ per volume; single issues, $\$ 1.25$.

Subscriptions, orders for back numbers, and changes of address should be sent to the publishers, University of California Press, Berkeley 4, California.

Printed at Ann Arbor, Michigan. Entered as second class matter at the Post Office, Berkeley, California.

* During the absence of E.G. Straus.

UNIVERSITY OF CALIFORNIA PRESS - BERKELEY AND LOS ANGELES 


\section{Pacific Journal of Mathematics}

\section{Vol. 5, No. $4 \quad$ December, 1955}

Richard Horace Battin, Note on the "Evaluation of an integral occurring in servomechanism theory" ............................. 481

Frank Herbert Brownell, III, An extension of Weyl's asymptotic law for eigenvalues................................. 483

Wilbur Eugene Deskins, On the homomorphisms of an algebra onto Frobenius algebras .................................. 501

James Michael Gardner Fell, The measure ring for a cube of arbitrary dimension ....................................... 513

Harley M. Flanders, The norm function of an algebraic field extension. II ............................................ 519

Dieter Gaier, On the change of index for summable series ............ 529

Marshall Hall and Lowell J. Paige, Complete mappings of finite groups . . . . 541

Moses Richardson, Relativization and extension of solutions of irreflexive relations..................................... 551

Peter Scherk, An inequality for sets of integers .................. 585

W. R. Scott, On infinite groups ........................... 589

A. Seidenberg, On homogeneous linear differential equations with arbitrary constant coefficients ......................................... 599

Victor Lenard Shapiro, Cantor-type uniqueness of multiple trigonometric integrals...

Leonard Tornheim, Minimal basis and inessential discriminant divisors for a cubic field...

Helmut Wielandt, On eigenvalues of sums of normal matrices ... 